$\S=-1$

\title{
Robotic Exoskeleton Control for Lower Limb Rehabilitation of Knee Joint
}

\author{
Syed Faiz Ahmed ${ }^{1 *}$, M. Kamran Joyo ${ }^{1}$, Athar Ali ${ }^{1}$, Abdul Malik M. Ali ${ }^{1}$, Kushsairy A. Kadir ${ }^{1}$, Yarooq R. Naqvi ${ }^{1}$, \\ Badri A. Bakar', Asadullah Shah² \\ ${ }^{\mathbf{1}}$ Universiti Kuala Lumpur- British Malaysian Institute Kuala Lumpur, Malaysia \\ ${ }^{2}$ International Islamic University Malaysia, KICT, Kuala Lumpur \\ *Corresponding authorE-mail: syedfaiz@unikl.edu.my
}

\begin{abstract}
Wearable devices such as exoskeletons are being opted frequently during rehabilitation processes for the post stroke recovery. Such devices are playing important role in the development of assistive rehabilitation robotic systems. In this paper three control strategies MPC and LQR and PID are introduced which were applied to knee joint of lower limb exoskeleton model for passive exercise. The two controls MPC and LQR are model based control which empowers them for stable responses. In this paper the analysis of robustness of control is done under the noisy and disturbance conditions. The results showed good performance of the exoskeleton model with the applied controls in the provided condition. In the future work the applied controls will be implemented on hardware.
\end{abstract}

\section{Introduction}

The leading cause of permanent disability worldwide is the stroke [1]. Impairments due to stroke causes the partial paralysis of one side of the body which disables the patients to perform any daily living activities [2]. With the help of therapy such as rehabilitation helps to recover the lost abilities [3]. In the past, lower limb rehabilitation procedures were completely applied manually by the therapist to the patients effected from stroke. Integrating robotics in to rehabilitation improved the process of recovery and made the process easier for the therapist. Integrating treadmill therapy with the rehabilitation process improved the walking capability of the patients effected with incomplete penalization of paraplegic and tetraplegic [4]. However, the execution of the process for longer period of time seemed to be difficult for the therapists. With the advancement in technology in recent years, robot-assisted therapy devices have huge impact over rehabilitation therapy. These assistive robots are used to either compensate lack of functionalities that patient cannot perform or to recover from impairment [5]. These systems might not be able to provide complete cure to impairment but should be able to enhance and improve the functionality of the impairments. The

Rehabilitation process consists of repetitive exercises which are designed to improve the motor functions. These exercises are either passive or active. In an active exercise, subjects put efforts to move their effected limbs while in passive exercise, patients are assisted by either therapist in a carefully designed pattern repetitively. The contribution of robotic technology in therapeutic exercises has opened new means for monitoring and training patients. For assisting the patients to do rehabilitation exercises, the assistive robots must be stable and robust enough to assist the motion freely in a repetitive and limited angular pattern. The robustness and stability come to the robot with the control system. Many researchers have applied different control strategies to assistive robots and made a positive progress. However, this area has more room for research.

There are numerous devices which implemented different control techniques to their systems. An assistive robot known as Physiotherabot used for therapeutic exercise for lower limb rehabilitation used Impedance and PID control strategy to adjust the motion of the robot [6]. In other research, position of the robot was controlled with the use of MPC control where author claims to successfully implement a specific task of lifting the leg of human body in lying down position [7]. Another author used iterative LQR control as a feedback controller in order to obtain the required accuracy [8]. Another researcher developed a comparison of ILQR and adaptive PD control for lower limb exoskeleton device.

In this paper, PID, MPC and LQR control is analyzed on a lower limb 1 DOF system (knee joint). The first section of the paper provides the related work which has been one previously in this area. Section two of this paper focuses on modeling and control of the knee joint. Third section discusses on the results of the system and in the final section is based on the conclusion and future work.

\section{Model and control}

This section of the paper introduces a model of 1-DOF exoskeleton and its control. The kinematics of the model is derived using $\mathrm{DH}$ parametrization. The transformation provides the properties of angular and translational movements of the exoskeleton as shown in Fig. 1. Fig. 2 represents the physical hardware design. The Lagrange's mechanics is then used to model rigid body dynamics of the robot exoskeleton. All that is required to get the equation of motion is to derive expressions for kinetic and potential energy. After mathematical evaluation of systems kinetic and potential energies, torque of the knee joint can be determined for specified 
angular position, velocity and accelerations using equation (1) which is the expression for Lagrange.

$\frac{d}{d t} \frac{\partial L}{\partial \dot{q}_{j}}-\frac{\partial L}{\partial \dot{q}_{j}}=\tau_{\mathbf{j}}$

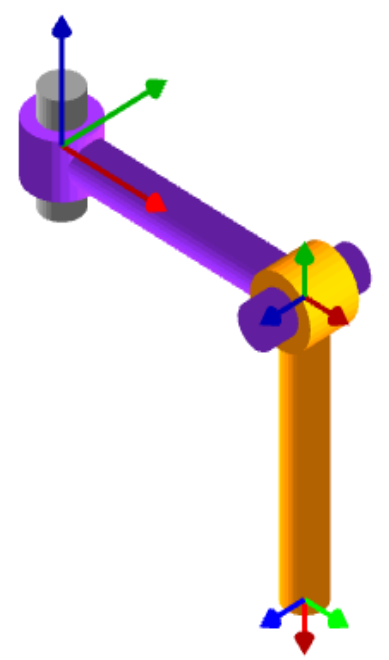

Fig. 1: Kinematic Model for Knee Joint

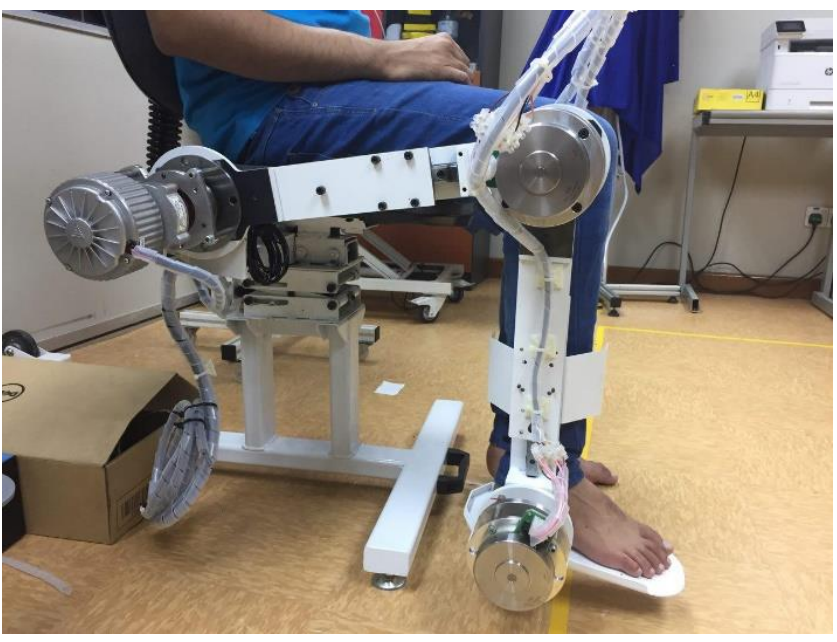

Fig. 2: Hardware design

The dynamic equation of the system is described by equation (2).

$\mathrm{D}(\theta) \theta+\mathrm{C}(\theta, \dot{\theta}) \ddot{\theta}+\mathrm{g}(\theta)=\tau_{\mathrm{L}}$

Where $D(\theta)$ is a inertial matrix robot manipulator, $C(\theta, \dot{\theta}) \dot{\theta}$ represents the Coriolis and centrifugal forces applied on the manipulator joint, $g(\theta)$ represents the gravitational vector. $\theta$ denotes the joint and angle and $\tau_{L}$ is the necessary torque to drive the knee joint.

Table 1: Parameters

\begin{tabular}{|c|c|c|}
\hline \multicolumn{3}{|c|}{ Design Parameters } \\
\hline Parameter & Symbols & Values \\
\hline Mass & $m$ & $2 \mathrm{~kg}$ \\
\hline Length & $l$ & $161.42 \mathrm{~cm}$ \\
\hline Height & $h$ & $21.28 \mathrm{~cm}$ \\
\hline \multicolumn{3}{|c|}{ Motor Parameter } \\
\hline $\mathrm{Ra}$ & \multicolumn{2}{c|}{0.50} \\
\hline $\mathrm{Kt}$ & 0.05 \\
\hline La & 0.23 \\
\hline Gear Ratio & 150 \\
\hline
\end{tabular}

Table I provides all the parameters required for mechanics and motor configuration. In this case, EC-90 motor parameters are used.

\section{Control system}

The control in passive mode is used to minimize the error based on the reference input. The system allows to perform certain movement to increase the ability of disabled patients to move their effected limbs. It handles the extension and flexion of the knee joint while the patient rests. A well-known PID control is used to generate the current required to move the motor to desired angle. The gain is fed to motor using equation (3). It tracks the trajectory using feedback controller by comparing the reference angle with the actual angle as sown in Fig. 3.

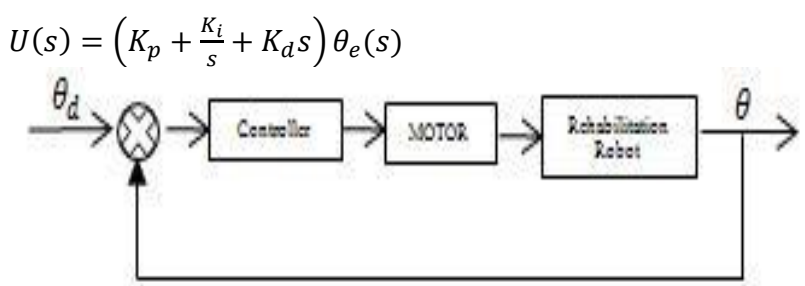

Fig. 3: Feedback control system for knee rehabilitation robot

Other controllers such as model predictive control and linear quadratic regulator were also applied to the same system. These two controllers differ in functionality with conventional PID as they require robots model to achieve the desired response. Their iterative process on the internal model makes sure that response of the system follows the reference provided to it. However, the response time and their action against any sensor noise or external disturbance is different. MPC controller uses Kalman to estimate the future states which enables it work better in noisy conditions. However, for faster response time MPC may be used to reject disturbance but not noise. Whereas LQR uses Kalman to estimate the future states of the system through its iterative process. This enables the controller to act better under noisy conditions but responds abruptly if disturbance is introduced to system.

To use MPC state space representation is required. As prediction and estimation process depends on Kalman. A detailed mathematical analysis of structure of MPC is given in the following article [9]. The state prediction process of the system is given by equation (4).

$\mathrm{x}(\mathrm{k})=\mathrm{Mx}(\mathrm{k})+\mathrm{Cu}(\mathrm{k})$

The optimization cost function is given by equation (5).

$\mathrm{J}(\mathrm{k})=\sum_{\mathrm{i}=0}^{\mathrm{N}-1}\left\{\mathrm{Q}\left[\mathrm{Z}^{\mathrm{T}}(\mathrm{k}+\mathrm{i} \mid \mathrm{k})-\mathrm{z}^{\mathrm{T}}(\mathrm{k}+\mathrm{N} \mid \mathrm{k})\right]^{2}+\mathrm{R}[\Delta \mathrm{u}(\mathrm{k}+\right.$ $\left.\mathrm{i} \mid \mathrm{k})]^{2}\right\}$

To use LQR, state space model is required which is derived using langrage's equation of motion. The state space feedback law is given by the equation (6).

$\mathrm{u}=-\mathrm{Kx}$

Which minimizes the cost function subject to system dynamics given by equation (7).

$x[n+1]=A x[n]+B u[n]$

$J=\sum_{n=0}^{\infty}\left[x^{T} Q_{x}+u^{T} R u+2 x^{T} N u\right]$

Equation (8) represents the cost function. To use LQR with the plant model, it is necessary to make sure that model is observable and controllable. Further mathematical analysis of LQR can be 
found in the following article [10-12]. Table II provides the control specifications used for all applied controls.

Table 2: Control Parameters

\begin{tabular}{|c|c|c|}
\hline \multicolumn{3}{|c|}{ Gain Parameters } \\
\hline \multirow{3}{*}{ Control } & Symbols & Values \\
\hline \multirow{3}{*}{ PID } & Proportional Gain $(P)$ & 2.7402 \\
\cline { 2 - 3 } & Integral Gain $(P)$ & 0.17492 \\
\cline { 2 - 3 } & Differential Gain $(P)$ & 8.757 \\
\hline \multirow{2}{*}{ MPC } & Prediction Horizon $(p)$ & 150 \\
\cline { 2 - 3 } & Control Horizon $(m)$ & 4 \\
\hline LQR & $K$ & \\
\hline
\end{tabular}

\section{Results}

The simulation tests of the controllers were performed in two uncertain conditions which is sensor noise and disturbance acting on the system. Fig. 3 represents the knee exoskeleton robot extension and flexion response with PID as a controller under disturbance. The applied rate of disturbance to the system varies from $10 \%$ to $100 \%$ and by the response, it can be realized that system has certain overshoot and steady state error.

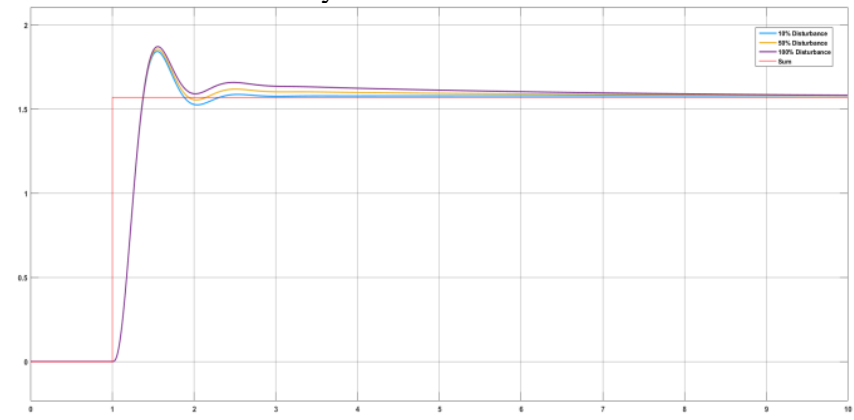

Fig. 3: PID response with different rate of applied disturbances

The response when introduced a noise in the system is shown in Fig.4

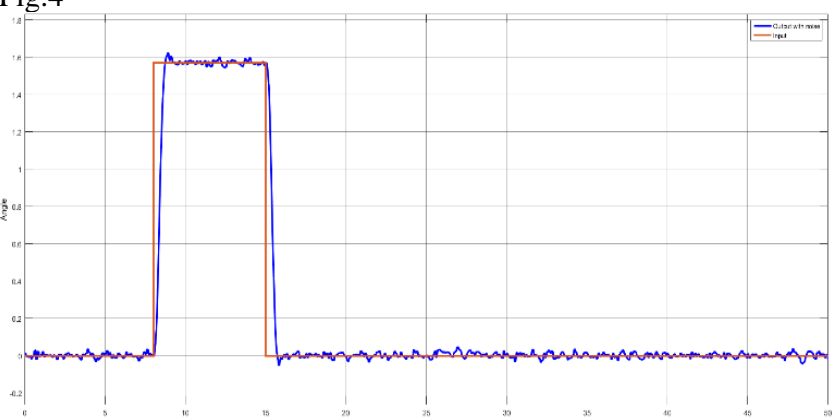

Fig. 4: Response of the system with PID when sensor noise is introduced

When switched to MPC, the system response with applied disturbance is shown in Fig.5. Fig. 6 represents the MPC response under noisy conditions by keeping the same control configurations as above.

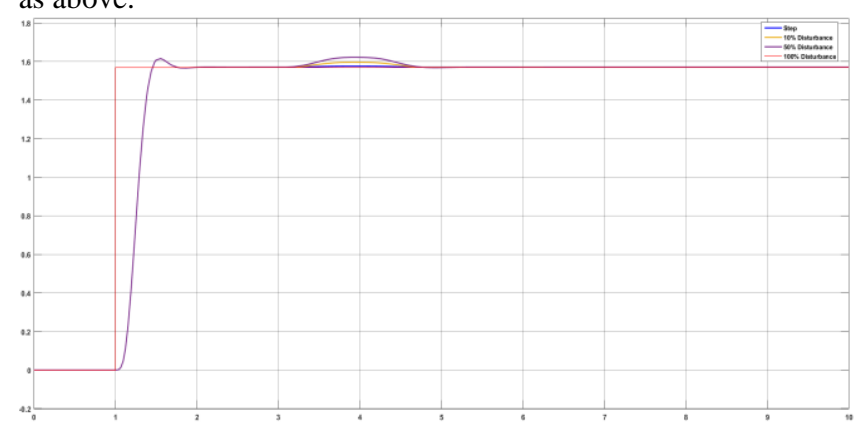

Fig. 5: MPC response with different rate of applied disturbances

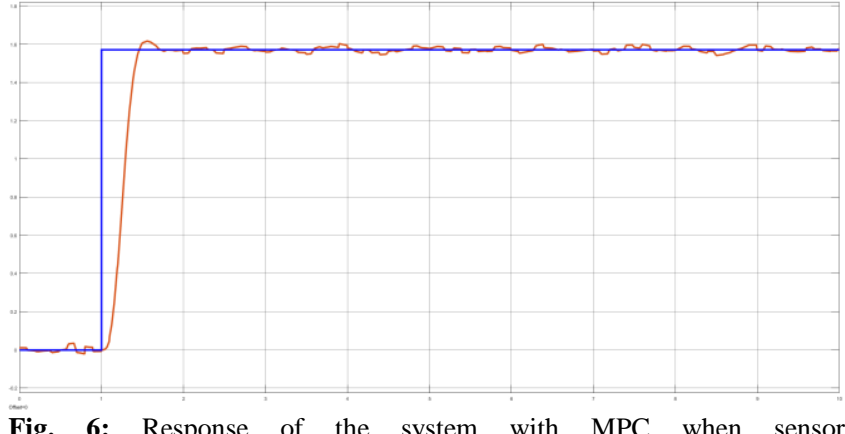

Fig. 6: Response of the system with MPC when sensor noise is introduced

Fig. 7 represents the LQR response under different rates of disturbance applied to the system without any sensor noise. While Fig. 8 represents the system response under noise and disturbance also. It can be realized that $\mathrm{LQR}$ is unable to control the response when disturbance is applied to the system while it responds to the sensor noise very well.

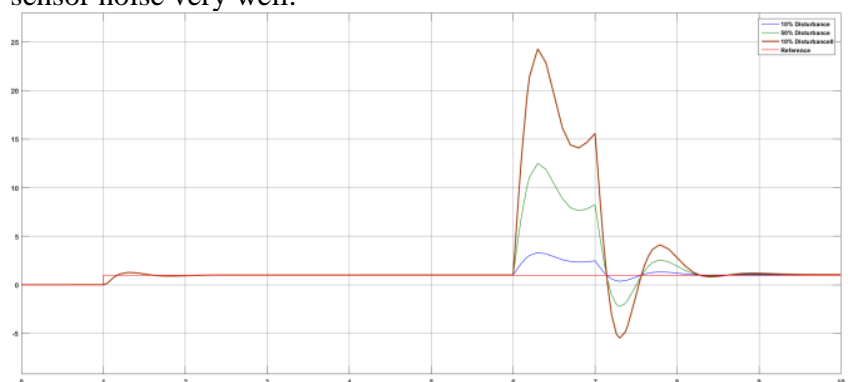

Fig. 7: LQR response with different rate of applied disturbances

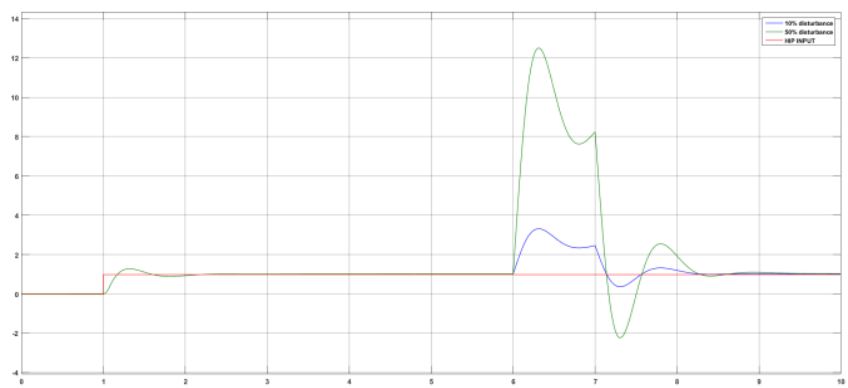

Fig. 8: Response of the system with MPC when sensor noise is introduced

The response of the system under different controllers provides analytical data on which comparison can be done. With PID, despite of overshoot system behaves well under disturbance applied to it. However, under noisy circumstances the system never settles to the desired angle as there exists a steady state error. With MPC, the same conditions are set, and system behaves better than PID as system overshoot is less and steady state error is controlled very quickly. With the introduction of noise in the system, it responds with uncontrollable steady state. With LQR also the same conditions are established, and it responds very well under noisy conditions but system response under disturbances gives a huge overshoot in the system.

\section{Conclusion}

Rehabilitation exoskeleton knee robotic system was developed and simulated under uncertain conditions. The two key factors to prove systems reliability are sensor noise and disturbance. In this paper, three different types controllers were implemented on the same system with same conditions. It was realized that PID produces an overshoot but settles the response quickly when disturbance is applied. While MPC produces less overshoot and under disturbances it quickly settles the response. Whereas LQR produces a huge overshoot under disturbance condition. When noise is 
introduced in the system, PID and MPC response is unsettled while LQR response is smoother. The actual task in future is to develop a control which can handle both factors at a time.

\section{References}

[1] D. Lloyd-Jones, R. J. Adams, T. M. Brown et al., "Heart disease and stroke statistics-2010 update: a report from the American heart association," Circulation, vol. 121, no. 7, pp. e46-e215, 2010.

[2] D. S. Smith, E. Goldenberg, A. Ashburn et al., "Remedial therapy after stroke: a randomised controlled trial," The British Medical Journal, vol. 282, no. 6263, pp. 517-520, 1981.

[3] M. Dam, P. Tonin, S. Casson et al., "The effects of long-term rehabilitation therapy on poststroke hemiplegic patients," Stroke, vol. 24, no. 8, pp. 1186-1191, 1993.

[4] Wernig, A.; Nanassy, A.; Muller, S. Maintenance of locomotor abilities following laufband (treadmill) therapy in para- and tetraplegic persons: Follow-up studies. Spinal Cord 1998, 36, 744-749.

[5] Dzahir MAM, Yamamoto S-I. Recent Trends in Lower-Limb Robotic Rehabilitation Orthosis: Control Scheme and Strategy for Pneumatic Muscle Actuated Gait Trainers. Robotics. 2014; 3(2):120-148

[6] Akdoğan, E., \& Adli, M. A. (2011). The design and control of a therapeutic exercise robot for lower limb rehabilitation: Physiotherabot. Mechatronics, 21(3), 509-522.

[7] Chow, Kevin, and Charles C. Kemp. "Robotic repositioning of human limbs via model predictive control." In Robot and Human Interactive Communication (RO-MAN), 2016 25th IEEE International Symposium on, pp. 473-480. IEEE, 2016.

[8] Ali, A., Ahmed, S. F., Joyo, M. K., \& Kushsairy, K. (2017, August). MPC-PID comparison for controlling therapeutic upper limb rehabilitation robot under perturbed conditions. In Engineering Technologies and Social Sciences (ICETSS), 2017 IEEE 3rd International Conference on (pp. 1-5). IEEE

[9] Jatsun, S., S. Savin, and A. Yatsun. "Motion control algorithm for a lower limb exoskeleton based on iterative LQR and ZMP method for trajectory generation." International Workshop on Medical and Service Robots. Springer, Cham, 2016.

[10] Tanveer, M. H., Hazry, D., Ahmed, S. F., Joyo, M. K., Warsi, F. A. Kamaruddin, H., ... \& Shahriman, A. B. (2014, March). NMPC-PID based control structure design for avoiding uncertainties in attitude and altitude tracking control of quad-rotor (UAV). In Signal Processing \& its Applications (CSPA), 2014 IEEE 10th International Colloquium on (pp. 117-122). IEEE.

[11] Joyo, M. K., Ahmed, S. F., Bakar, M. I. A., \& Ali, A. (2018). Horizontal Motion Control of Underactuated Quadrotor Under Disturbed and Noisy Circumstances. In Information and Communication Technology (pp. 63-79). Springer, Singapore.

[12] Ahmed, S. F. (2007, December). A new approach in Industrial automation application" Embedded system design for Injection Molding Machine". In Multitopic Conference, 2007. INMIC 2007. IEEE International (pp. 1-5). IEEE

[13] Ahmed, S. F., Kushsairy, K., Bakar, M. I. A., Hazry, D., \& Joyo, M. K. (2015, April). Attitude stabilization of Quad-rotor (UAV) system using Fuzzy PID controller (an experimental test). In Computing Technology and Information Management (ICCTIM), 2015 Second International Conference on (pp. 99-104). IEEE. 